\begin{tabular}{|l|l|l||}
\hline \multicolumn{2}{|c|}{ PublisherInfo } \\
\hline \hline PublisherName & $:$ & BioMed Central \\
\hline \hline PublisherLocation & $:$ & London \\
\hline \hline PublisherImprintName & $:$ & BioMed Central \\
\hline \hline
\end{tabular}

\title{
Investigation of B cell auto-reactivity in Fas-deficient mice
}

\begin{tabular}{|l|l|l||}
\hline \multicolumn{2}{|c|}{ ArticleInfo } \\
\hline \hline ArticleID & $:$ & 42 \\
\hline \hline ArticleDOI & $:$ & $10.1186 /$ ar-1999-66742 \\
\hline \hline ArticleCitationID & $:$ & 66742 \\
\hline \hline ArticleSequenceNumber & $:$ & 38 \\
\hline \hline ArticleCategory & $:$ & Paper Report \\
\hline \hline ArticleFirstPage & $:$ & 1 \\
\hline \hline ArticleLastPage & $:$ & 3 \\
\hline \hline & & RegistrationDate : 1999-11-4 \\
\hline ArticleHistory & $:$ & OnlineDate \\
\hline \hline ArticleCopyright & $:$ & Current Science Ltd1999-11-4 \\
\hline \hline ArticleGrants & $:$ & \\
\hline \hline ArticleContext & $:$ & 130752211 \\
\hline \hline
\end{tabular}




\section{Keywords}

Autoantibody, autoimmunity, B cell tolerance, Fas, rheumatoid factor

\section{Context}

High titre autoantibodies are characteristic of a number of systemic autoimmune diseases. This paper investigated the regulation of autoreactive B cells in animals which have ongoing autoimmunity, and speculates how such autoreactive B cells are controlled in normal animals. The autoantigen investigated is self IgG2a rheumatoid factor (RF). The group has developed a transgenic (tg) model of B cell autoimmunity based on RF idiotype AM14. This idiotype was originally isolated from an MRL/fas/lpr mouse model of systemic lupus erythematosus, which has an apoptotic defect, due to the absence of Fas expression. As AM14 recognises only IgG2a of the a allotype (IgG2a/a), the authors have generated B cell transgenic mice (expressing AM14) which either express or lack the autoantigen (ie the a allotype). These animals were also made on both normal or autoimmune-prone backgrounds. The authors have previously shown that tg AM14 B cells are clonally ignorant in normal Balb/c mice, being neither tolerised nor activated in the presence of the autoantigen. Results presented here investigate the fate of autoreactive B cells in autoimmune-prone animals using C57B6/MRL/fas/lpr mice (B6/lpr). B6/lpr mice produce large numbers of autoantibodies and have mild autoimmune disease due chiefly to the Fas deficiency. RF AM14 tg mice were made by crossing the separate heavy $(\mathrm{H})$ and light $(\mathrm{L})$ chain transgenes that encode the AM14 RF specificity on to both B6/lpr/IgHa(autoantigen positive) and B6/ lpr/IgHa (autoantigen negative) backgrounds. These congenic B cell tg B6/lpr strains were used to study the activation/regulation of RF B cells and T cells in vivo. To test the hypothesis that autoreactive B cells are not necessarily tolerised in normal mice, and that such B cells become selectively activated in autoimmune animals. The authors investigated whether B cell activation was autoantigen driven, or was due to polyclonal activation. In addition, the authors investigated the role of Fas in regulating autoreactive B cells.

\section{Significant findings}

Age matched cohorts of four groups of AM14 tg mice were studied: $\mathrm{H}$ and L tg mice on B6/lpr background, $\mathrm{H}$ chain only tg on B6/lpr background, non tg mice on B6/lpr background and $\mathrm{H}$ and $\mathrm{L}$ tg mice on the non autoimmune Balb/c background. All four groups were established on $\operatorname{IgHa}$ and $\operatorname{IgHb}$ backgrounds to test the effect of presence or absence of autoantigen. The authors showed that the total numbers of spleen cells were greater in both $\mathrm{H}$ and $\mathrm{L}$, and $\mathrm{H}$ tg mice on B6/lpr in the presence of IgHa autoantigen. In addition percentages and numbers of AM14 idiotype positive B cells were greater in $\mathrm{H}$ 
and $\mathrm{L}$, and $\mathrm{H}$ chain tg mice on B6/lpr background in the presence of autoantigen. No differences were seen in non tg mice in the presence or absence or autoantigen. No differences were were seen in $\mathrm{H}$ and $\mathrm{L}$ $\operatorname{tg}$ mice on a Balb/c background in the presence or absence of autoantigen. A substantial fraction of the idiotype positive cells were thought to be activated as shown by increased expression of CD44. These cells were also thought to be differentiated into plasma cells, as detected in idiotype-specific ELISpot assays. Immunohistochemical analyses of spleen demonstrated large numbers of idiotype positive cells in the red pulp in AM14 tg, Fas-deficient, IgHa mice - an area known to harbour plasma cells early after immunisation in normal mice. T cell activation was determined by measuring $\mathrm{B} 220^{+} \mathrm{T}$ cells. Both $\mathrm{B} 220^{+} \mathrm{T}$ cell percentages and absolute numbers were increased in mice which expressed both $\mathrm{H}$ and $\mathrm{L}$ chain tg on the B6/lpr IgHa background. This was not seen in non tg mice or in tg mice on the Balb/c background. The increase in $\mathrm{T}$ cell numbers was dependent on the presence of $\mathrm{B}$ cell autoantigen.

\section{Comments}

The origin of autoreactive B cells is unclear and many groups have speculated on different pathways including cells that aberrantly escape central tolerance, cells that are abnormally rescued from an anergic state, or cells that are derived from clonally ignorant B cells whose activation/tolerisation is improperly regulated. This interesting model sugests that B cells are clonally ignorant in a non autoimmune animal, but are driven to expansion, activation and autoantibody production in an autoimmunity-prone, Fas-deficient animal. The model should provide unique opportunities to study the regulation of defined, non tolerant, mature B cells and their effect on autoreactive T cells. This paper's extensive discussion may also provide a useful overview of some of the controversies in this field.

\section{Methods}

Three strains of tg mice were constructed: AM14 Vh/B6/lpr(H chain tg), Vk/B6/lpr and AM14 Vk/ B6/lpr/IgHa (L chain tgs). At back-cross four to the B6/lpr genetic background, these three strains were intercrossed to create antigen-positive double tg mice or antigen-negative double tg mice. Mice were analysed at 4-7 months of age, after the onset of spontaneous autoimmunity. Spleens were harvested, weighed and divided into two portions. One portion was quick frozen for histochemical analysis. The other portion was processed into a single cell suspension for cell number analysis or fluorescenceactivated cell sorter analysis.

\section{References}

1. Wang H, Shlomchik MJ: Autoantigen-specific B cell activation in Fas-deficient rheumatoid factor immunoglobulin transgenic mice. J Exp Med. 1999, 190: 639-649.

This PDF file was created after publication. 